Published in final edited form as:

Nat Methods. 2014 August ; 11(8): 783-784. doi:10.1038/nmeth.3047.

\title{
Improved vectors and genome-wide libraries for CRISPR screening
}

\author{
Neville E. Sanjana ${ }^{\# 1,2,3,4}$, Ophir Shalem $\# 1,2,3,4$, and Feng Zhang ${ }^{1,2,3,4}$ \\ ${ }^{1}$ Broad Institute of MIT and Harvard 7 Cambridge Center Cambridge, MA 02142, USA \\ ${ }^{2}$ McGovern Institute for Brain Research Massachusetts Institute of Technology Cambridge, MA \\ 02139, USA \\ ${ }^{3}$ Department of Brain and Cognitive Sciences Massachusetts Institute of Technology Cambridge, \\ MA 02139, USA \\ ${ }^{4}$ Department of Biological Engineering Massachusetts Institute of Technology Cambridge, MA \\ 02139, USA \\ \# These authors contributed equally to this work.
}

\begin{abstract}
Genome-wide, targeted loss-of-function pooled screens using the CRISPR (clustered regularly interspaced short palindrome repeats)-associated nuclease Cas9 in human and mouse cells provide an alternative screening system to RNA interference (RNAi) and have been used to reveal new mechanisms in diverse biological models ${ }^{1-4}$. Previously, we used a Genome-scale CRISPR Knock-Out (GeCKO) library to identify loss-of-function mutations conferring vemurafenib resistance in a melanoma model ${ }^{1}$. However, initial lentiviral delivery systems for CRISPR screening had low viral titer or required a cell line already expressing Cas9, limiting the range of biological systems amenable to screening.
\end{abstract}

Here, we sought to improve both the lentiviral packaging and choice of guide sequences in our original GeCKO library ${ }^{1}$, where a pooled library of synthesized oligonucleotides was cloned into a lentiviral backbone containing both the Streptococcus pyogenes Cas9 nuclease and the single guide RNA (sgRNA) scaffold. To create a new vector capable of producing higher-titer virus (lentiCRISPRv2), we made several modifications, including removal of one of the nuclear localization signals (NLS), human codon-optimization of the remaining NLS and P2A bicistronic linker sequences, and repositioning of the U6-driven sgRNA cassette (Fig. 1a). These changes resulted in a $\sim 10$-fold increase in functional viral titer over lentiCRISPRv $1^{1}$ (Fig. 1b).

\footnotetext{
zhang@broadinstitute.org

Author Contributions

N.S., O.S., and F.Z. conceived and designed the experiments. N.S. and O.S. performed the experiments and analyzed the data. N.S., O.S., and F.Z. wrote the manuscript.

Competing Financial Interests

A patent application has been filed relating to this work, and the authors plan on making the reagents widely available to the academic community through Addgene and to provide software tools via the Zhang lab website (http://www.genome-engineering.org/). F.Z. is a scientific advisor for Editas Medicine.
} 
To further increase viral titer, we also cloned a two-vector system, in which Cas9 (lentiCas9-Blast) and sgRNA (lentiGuide-Puro) are delivered using separate viral vectors with distinct antibiotic selection markers (Fig. 1a). LentiGuide-Puro has a 100-fold increase in functional viral titer over the original lentiCRISPRv1 (Fig. 1b). Both single and dual-vector systems mediate efficient knock-out of a genomically-integrated copy of EGFP in human cells (Supplementary Fig. 1). Whereas the dual vector system enables generation of Cas9-expressing cell lines which can be subsequently used for screens using lentiGuidePuro, the single vector lentiCRISPRv2 may be better suited for in vivo or primary cell screening applications.

In addition to the vector improvements, we designed and synthesized new human and mouse GeCKOv2 sgRNA libraries (Supplementary Methods) with several improvements (Table 1): First, for both human and mouse libraries, to target all genes with a uniform number of sgRNAs, we selected 6 sgRNAs per gene distributed over 3-4 constitutively expressed exons. Second, to further minimize off-target genome modification, we improved the calculation of off-target scores based on specificity analysis ${ }^{5}$. Third, to inactivate microRNAs (miRNAs) which play a key role in transcriptional regulation, we added sgRNAs to direct mutations to the pre-miRNA hairpin structure ${ }^{6}$. Finally, we targeted $\sim 1000$ additional genes not included in the original GeCKO library.

Both libraries, mouse and human, are divided into 2 sub-libraries — containing 3 sgRNAs targeting each gene in the genome, as well as 1000 non-targeting control sgRNAs. Screens can be performed by combining both sub-libraries, yielding 6 sgRNAs per gene, for higher coverage. Alternatively, individual sub-libraries can be used in situations where cell numbers are limiting (eg. primary cells, in vivo screens). The human and mouse libraries have been cloned into lentiCRISPRv2 and into lentiGuide-Puro and deep sequenced to ensure uniform representation (Supplementary Fig. 2, 3). These new lentiCRISPR vectors and human and mouse libraries further improve the GeCKO reagents for diverse screening applications. Reagents are available to the academic community through Addgene and associated protocols, support forums, and computational tools are available via the Zhang lab website (www.genome-engineering.org).

\section{Supplementary Material}

Refer to Web version on PubMed Central for supplementary material.

\section{Acknowledgements}

We also would like to thank the entire Zhang Lab for support and helpful advice. N.S. is supported by a postdoctoral fellowship from the Simons Center for the Social Brain at MIT. O.S. is a Klarman Fellow of the Broad-Israel Partnership. F.Z. is supported by the NIH and NIMH through a Director's Pioneer Award (5DP1MH100706), a Transformative R01 grant (5R01-DK097768), the Keck, Merkin, Vallee, Damon Runyon, Searle Scholars, Klingenstein, and Simons Foundations, Bob Metcalfe, and Jane Pauley.

\section{References}

1. Shalem O, et al. Science. 2014; 343:84-87. [PubMed: 24336571]

2. Wang T, Wei JJ, Sabatini DM, Lander ES. Science. 2014; 343:80-84. [PubMed: 24336569] 
3. Koike-Yusa H, Li Y, Tan E-P, Velasco-Herrera MDC, Yusa K. Nat Biotechnol. 2013; 32:267-273. [PubMed: 24535568]

4. Zhou Y, et al. Nature. 2014; 509:487-491. [PubMed: 24717434]

5. Hsu PD, et al. Nat Biotechnol. 2013; 31:827-832. [PubMed: 23873081]

6. Zhao Y, et al. Sci Rep. 2014; 4:3943. [PubMed: 24487629] 
a

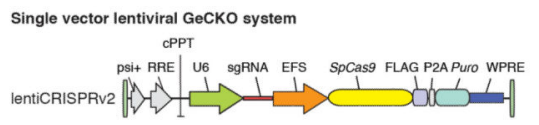

Dual vector lentiviral GeCKO system
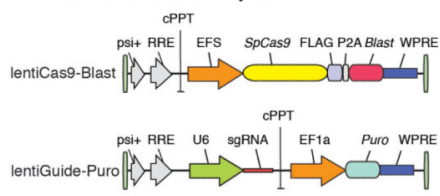

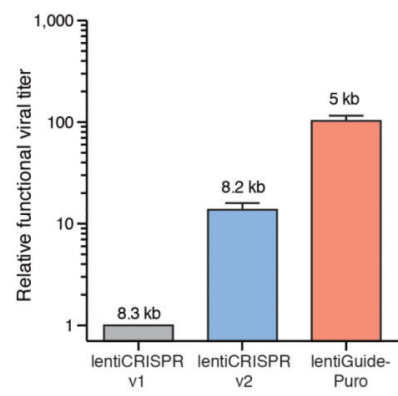

Figure 1.

New lentiviral CRISPR designs produce viruses with higher functional titer.

(a) Lentiviral expression vector for Streptococcus pyogenes Cas9 and sgRNA in the improved one vector system (lentiCRISPR v2) and the two vector system (lentiCas9-Blast, lentiGuide-Puro). Psi packaging signal (psi+), rev response element (RRE), central polypurine tract (cPPT), elongation factor-1a short promoter (EFS), FLAG octapeptide tag (FLAG), 2A self-cleaving peptide (P2A), puromycin selection marker (puro), posttranscriptional regulatory element (WPRE), blasticidin selection marker (blast), and elongation factor-1a promoter (EF1a). (b) Relative functional viral titer of viruses made from lentiCRISPR v $1^{1}$, lentiCRISPR v2, and lentiGuide-Puro vector with a EGFP-targeting sgRNA ( $n=3$ independently-transfected virus batches with 3 replicate transductions per construct). HEK293FT cells were transduced with serial dilutions of virus and, after 24 hours, selected using puromycin $(1 \mathrm{ug} / \mathrm{ml})$. Puromycin-resistant cells were measured after 4 days from the start of selection using the CellTiter-Glo (Promega) luciferase assay. Relative titers were calculated using viral volumes that yielded less than $20 \%$ puromycin-resistant cells in order to minimize the number of cells with multiple infections. Numbers above each bar indicate the size of the packaged virus for each construct. 


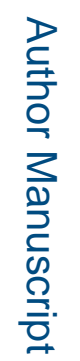

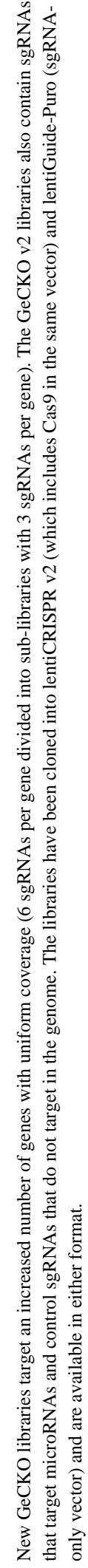

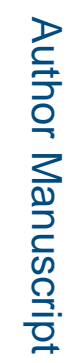

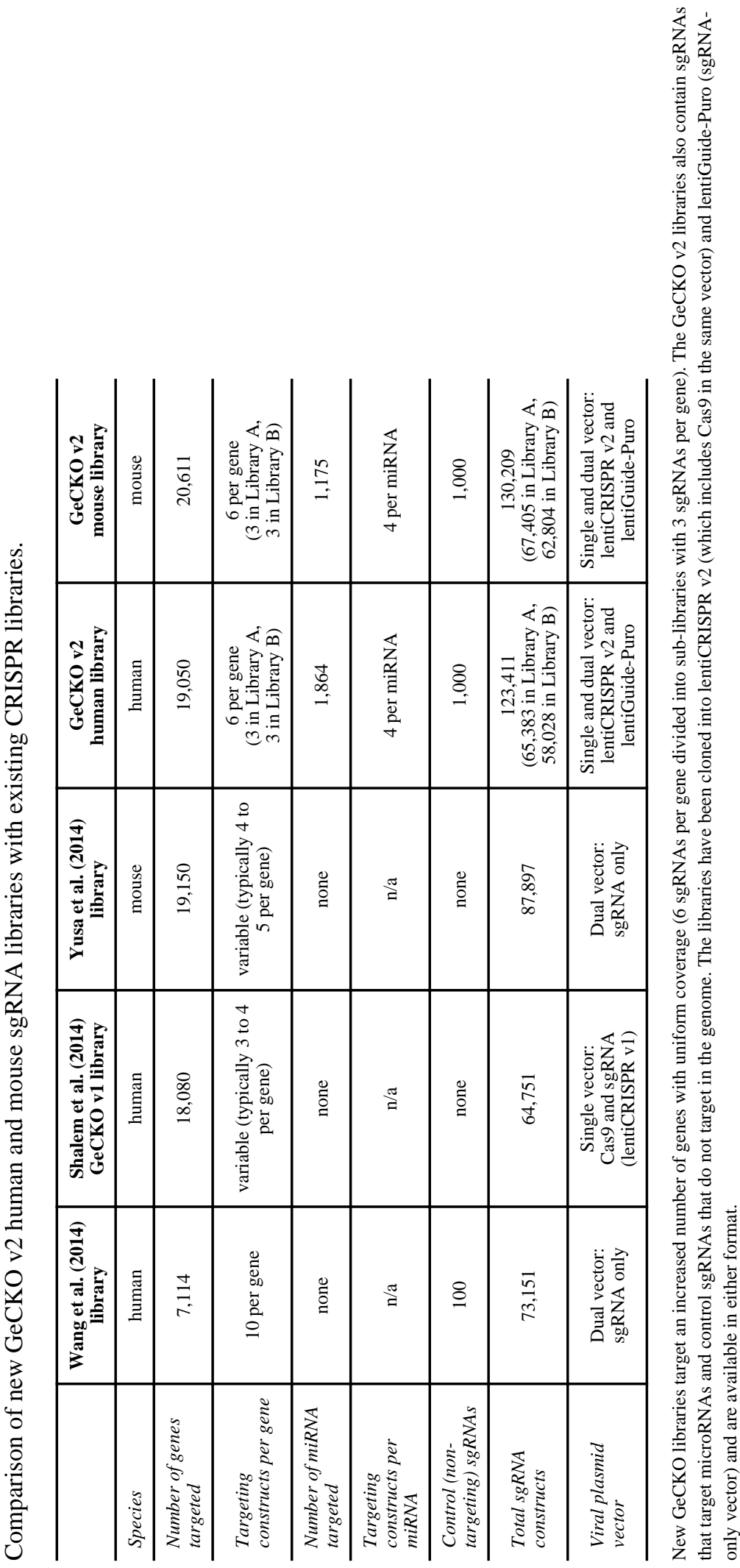

Nat Methods. Author manuscript; available in PMC 2015 June 30. 